lesions occurring primarily in children and adolescents. Symptoms of presentation may range from mild unspecific bone pain, local swelling and warmth to severe pain, malaise, fevers and even fractures.

Objectives: In this study, we aimed to evaluate our patients who had a diagnosis of CRMO, retrospectively.

Methods: Six patients who were diagnosed with CRMO between 2010-2017 years were included in the study. The CRMO diagnosis was based on characteristic clinical features and magnetic resonance imaging findings. The clinical data were obtained from the records of electronic files.

Results: The female to male ratio of the cases was $4 / 2$ and the median age was 11.15 years. $^{6-12}$ The age of diagnosis was 10.35 years $(4-12.5)$, the median period for diagnosis delay was 3 years $(0.75-8)$. The most common complaint was localised pain ( $n=6,100 \%)$. Accompanying diseases were detected in 3 patients; 1 case had inflammatory myositis, 1 case had PFAPA syndrome and 1 case had selective IgA deficiency. Multifocal bone involvement was present in 4 $(66 \%)$ cases and unifocal bone involvement in $2(33 \%)$ cases. The most common site of disease was femur. Acute phase reactants were high most of the cases; elevated erythrocyte sedimentation rate $(E S R)$ in 5 cases $(83.3, n=6)$, elevated creactive protein level in 4 cases $(66.6 \%, n=6)$, elevated serum amyloid a level in 3 cases $(60 \%, n=5)$, and elevated fibrinogen in 2 cases $(50 \%, n=4)$ were present. ANA was found positive at low titer in only 1 case, whereas rheumatoid factor was negative in all cases. Non-steroidal anti-inflammatory drugs were prescribed in all cases and anti TNF drugs in 3 (Etanercept in 2 cases and adalimumab in 1 case). Clinical characteristics of the patients are given in Table 1.

Abstract AB1121 - Table 1. Clinical findings of the cases Initial complaints of cases

\begin{tabular}{lcc}
\hline & $\mathrm{n}$ & $\%$ \\
\hline Pain & 6 & 100 \\
Walking abnormality & 4 & 66.7 \\
Swelling on bone & 1 & 16.7 \\
Weight loss & 1 & 16.7 \\
Distribution of involved bones & & \\
Femur & 4 & 66.7 \\
Iliac bone & 2 & 33.3 \\
Tibia & 2 & 33.3 \\
Calcaneus & 1 & 16.7 \\
Vertebral column & 1 & 16.7 \\
Acetabulum & 1 & 16.7 \\
Treatment & & \\
lbuprofen & 3 & 50 \\
Naproxen Na & 2 & 33.3 \\
Aspirin & 1 & 16.7 \\
Indomethacin & 1 & 16.7 \\
Adalimumab & 1 & 16.7 \\
Etanercept & 2 & 33.3 \\
\hline
\end{tabular}

Conclusions: The diagnosis of CRMO is difficult and no consensus exist on diagnosis and treatment. Multifocal bone lesions with characteristic radiological findings are very suggestive of CNO. The first line treatment is usually NSAIDs, however, anti TNF treatment are needed in some patients to achieve for remission. Our case is the second one who had inflammatory myositis and CRMO according the literature.

Disclosure of Interest: None declared

DOI: 10.1136/annrheumdis-2018-eular.3104

\section{AB1122 DIALYSIS TREATMENT OF SYSTEMIC VASCULITIS IN PAEDIATRIC PATIENTS}

P. Miteva ${ }^{1}$, D. Roussinov ${ }^{1}$, R. Shumnalieva ${ }^{2}$, S. Monov ${ }^{2} .{ }^{1}$ Department of Pediatrics, Clinic of Pediatric Nephrology and Dialysis; ${ }^{2}$ Department of Internal Medicine, Clinic of rheumatology, Medical University - Sofia, Sofia, Bulgaria

Background: Childhood-onset systemic vasculitis is a rare but serious condition with high mortality rate even with proper treatment. Renal involvement at presentation is a high risk for end stage renal disease (ESRD).

Objectives: The aim of our study was to review the results of the dialysis treatment in paediatric patients with systemic vasculitis in a single dialysis centre for children in Bulgaria.

Methods: For a period of 20 years we observed 9 clinical cases of systemic vasculitis -5 cases of Goodpasture syndrome (GPS) -4 girls and 1 boy from 6 to 17 years old; one 6 year old girl and one 12 year old boy with Granulomatosis with polyangitis (GPA); an 8 month old boy with microscopic polyangiitis (mPAN), and one 8 year old girl with Takayasu arteritis. Seven of the children were treated by hemodialysis because of progressive kidney failure leading to ESRD, one of them was treated by continuous ambulatory peritoneal dialysis (CAPD) and one conservatively.
Results: In the GPS group one of the girls still continues on hemodialysis with no pulmonary symptoms for more than 14 years follow-up. The second girl was treated with immunoadsorption after which reminded antibody negative. On the follow-up she is managed conservatively for chronic kidney disease. Two of the girls died because of severe pulmonary bleeding caused by exposure to fragran smoke during incense. The boy was treated conservatively because of mild pulmonary and kidney involvement and died from pulmonary bleeding caused by smoke. In the GPA group the girl underwent kidney transplantation and died one month later, and the boy died because of severe pulmonary bleeding. The 8 month old boy with mPAN was treated by dialysis with some clinical improvement but after six months died because of thrombus in the right atrium. The girl with Takayasu arteritis was treated with CAPD but died because of cardiopulmonary complications.

Conclusions: End stage renal disease is poor prognostic factor for survival in paediatric patients with systemic vasculitis. $75 \%$ of the children died in a period of 5 months after initiation of dialysis treatment.

Disclosure of Interest: None declared

DOI: 10.1136/annrheumdis-2018-eular.5836

\section{AB1123 \\ DO CHILDREN WITH JUVENILE IDIOPATHIC ARTHRITIS PLAY AN ACTIVE ROLE IN THEIR TREATMENT ADHERENCE? FIRST RESULTS OF THE RUMAJI STUDY}

G. MONTAGU ${ }^{1}$, E. Mevel ${ }^{1}$, L. Rossi Semerano ${ }^{2}$, E. Solau Gervais ${ }^{3}$, S. Trope ${ }^{4}$, J.D. Cohen ${ }^{5}$, on behalf of ANDAR patient organisation. ${ }^{1}$ Sociology and research, Unknowns; ${ }^{2}$ Kremlin Bicêtre Hospital, Paris; ${ }^{3}$ Hospital, Poitiers; ${ }^{4}$ ANDAR, PARIS ${ }^{5}$ Hospital, Montpellier, France

Background: Adherence to DMARDs such as methotrexate and biologics is critical for patients with Juvenile Idiopathic Arthritis (JIA). Notwithstanding, few stud ies exists on that topic and we lack information to understand the grounds for adherence.

Objectives: The RUMAJI study aims, among others, to understand and deciphe the parents and children adherence mechanisms and practices.

Methods: Qualitative methods were chosen in order to investigate parents' and children's everyday life with JIA and its treatment. An ethnographic study was designed by a multidisciplinary team including rheumatologists, paediatricians, patient associations members and anthropologists. The study involved 15 families (enough to reach saturation), recruited from 5 centres by diversity of clinical and sociological profiles. The panel included 17 children with JIA, 11 girls and 6 boys median age $10,{ }^{3 ;} 17$ median disease duration $2.5 .^{1 ; 15} 4$ children were treated with conventional DMARDs in monotherapy, 4 with biologic DMARDs in monotherapy 5 with cDMARD-bDMARD association and 4 with NSAIDs only.

Interviews were conducted by anthropologists at family's home using in-depth semi directive and biographic methods. 3 fields were explored: organisation of everyday life with JIA, treatment practices, impact on school and social activities. Interviews were recorded and transcribed for analysis.

Results: Adherence results from an appropriation process of the JIA and treatment that require both an active role from parents and children, even before the transition. This active role played by children could be either stimulated or inhibited at home according to the family's structure, social background and parents attitudes toward their child (participation to the decision, explanation of the disease)

Children's active role includes in particular: 1) negotiations with parents and physician, 2) experiments with the treatment (forgetting or involuntary switch from the parents, changing the dosage on their own initiative) and 3) participation to the treatment administration and ritualization.

The manner children consider and manage their DMARDs is the result of an arbitration depending on the positive (a) and side effects (b) they felt in their body and the effects noted by the doctors (c) during the examinations and test results. Dealing with these 3 dimensions requires to link together both a theoretical and practical knowledge of JIA. Thus, children build their own and singular knowledge of their disease and treatment, which is a source of control of their body and their life. Conclusions: Qualitative methods, through an ethnographic study starting from children's point of view, underline the active role they play in their care. Adherence to DMARDs could be improved by supporting children's implication as soon as the beginning of JIA.

Acknowledgements: This work was supported by an institutionnal grant from NORDIC Pharma to ANDAR. All medical and patient experts volunteers.

Disclosure of Interest: None declared

DOI: 10.1136/annrheumdis-2018-eular.5499 